RACAR : Revue d'art canadienne

Canadian Art Review

RACAR

\title{
Pour voir le passé d'un tableau : les tableaux vivants de l'Allégorie du ministre parfait d'Eustache Le Sueur (1653) dans le film Une disparition (2017). Entretien avec Éric Méchoulan et Marion Froger
}

\section{Ersy Contogouris}

Volume 44, numéro 2, 2019

Stay Still: Past, Present, and Practice of the Tableau Vivant

Stay Still : histoire, actualité et pratique du tableau vivant

URI : https://id.erudit.org/iderudit/1068326ar

DOI : https://doi.org/10.7202/1068326ar

Aller au sommaire du numéro

Éditeur(s)

UAAC-AAUC (University Art Association of Canada | Association d'art des universités du Canada)

ISSN

0315-9906 (imprimé)

1918-4778 (numérique)

Découvrir la revue

Citer ce document

Contogouris, E. (2019). Pour voir le passé d'un tableau : les tableaux vivants de l'Allégorie du ministre parfait d'Eustache Le Sueur (1653) dans le film Une disparition (2017). Entretien avec Éric Méchoulan et Marion Froger. RACAR : Revue d'art canadienne / Canadian Art Review, 44(2), 175-182.

https://doi.org/10.7202/1068326ar

Tous droits réservés (C) UAAC-AAUC (University Art Association of Canada | Association d'art des universités du Canada), 2019
Ce document est protégé par la loi sur le droit d'auteur. L'utilisation des services d'Érudit (y compris la reproduction) est assujettie à sa politique d'utilisation que vous pouvez consulter en ligne. 


\title{
Pour voir le passé d'un tableau: les tableaux vivants de l'Allégorie du ministre parfait d'Eustache Le Sueur (1653) dans le film Une disparition (2017). Entretien avec Éric Méchoulan et Marion Froger
}

\author{
Ersy Contogouris
}

Éric Méchoulan est professeur au département des littératures de langue française à l'Université de Montréal. Ses recherches actuelles portent sur les sentiments politiques à l'âge classique, sur l'histoire intermédiale des idées, sur les archives et la mémoire, ainsi que sur le temps qui passe. Il a fondé et dirigé la revue Intermédialités et a été directeur du Centre de Recherches Intermédiales sur les arts, les lettres et les techniques (CRlalt) de 2009 à 2013.

-eric.mechoulan@umontreal.ca

Marion Froger est professeure au département d'histoire de l'art et d'études cinématographiques de I'Université de Montréal. Elle s'intéresse à la part du cinéma dans la formation du lien social, sur une scène imaginaire (celle qui permet au spectateur de se lier aux personnes filmées et aux filmeurs) comme sur le terrain concret des pratiques cinématographiques. Elle dirige la revue Intermédialités depuis 2013.

-marion.froger@umontreal.ca

Ersy Contogouris est professeure adjointe d'histoire de l'art à l'Université de Montréal.

-ersy.contogouris@umontreal.ca
ERSY CONTOGOURIS Votre court-métrage Une disparition. Enquête sur un tableau de cheminée d'Eustache Le Sueur (https://vimeo.com/212632352), que vous avez conçu et réalisé, et dans lequel Marion Froger figure en tant qu'Athéna / la Sagesse, se présente comme une réflexion sur le tableau Allégorie du ministre parfait exécuté par le peintre classique français Eustache Le Sueur en 1653. | fig. 1 | Le film débute avec la question suivante: "Quelle(s) perspective(s) choisir pour voir le passé de ce tableau?». Le film d'une vingtaine de minutes se déploie ensuite comme une série de réflexions à partir de divers points de vue, dont plusieurs sont des tableaux vivants. Mais la première reconstitution du tableau est performée par une femme devant une bibliothèque de livres anciens, qui manipule des objets-une théière pour le Conseil, un presse-agrumes pour la Sagesse et un cendrier avec une cuiller pour la Prudence-en expliquant la signification de chaque objet. | fig. 2 |

ÉRIC MÉCHOULAN Il me paraissait important de commencer, pour la première fois où on voit le tableau, justement avec des objets, puisque le tableau est une allégorie. Le principe de l'allégorie est de prendre n'importe quoi et dire: «c'est la Prudence ou la Sagesse». Il y a des codes qui ont fait qu'Athéna va représenter la Sagesse, ou que la Prudence va tenir un miroir. On peut mettre le Conseil ou une théière, si je vous dis «ceci est le Conseil», alors c'est le Conseil, point final. Commencer avec des objets accessoires était une manière de dire qu'ils sont infiniment plus importants qu'ils n'en ont l'air. Ils peuvent même représenter la Sagesse, la Prudence. L'accessoire devient alors le sujet.

EC Le terme «tableau vivant» apparaît en contraste à «nature morte», mais cette opposition semble problématisée par cette mise en scène dans le film.

Éм En effet, puisqu'on a des objets, on pourrait dire que c'est une nature morte. Mais cet agencement est aussi un tableau vivant, avec des objets à la place de corps: peu importe, fondamentalement, que ce soit Marion en Athéna, ou mon presse-oranges, c'est pareil. Et il me semble que parce que ces objets ont des significations allégoriques, il ne s'agit plus d'une nature morte. De plus, les objets sont amenés: si j'avais simplement mis sur mon bureau les quatre "personnages» ou quatre objets sur des vieux livres et que la narratrice énonçait «voilà, c'est le tableau», on se serait rapproché du genre de la nature morte. Mais le fait de les faire apparaître un à la fois dans une animation très concrète et de les présenter par un discours oral («ceci est le Conseil»; «ceci est la Prudence», etc.) confère un aspect qui tend davantage vers le tableau vivant que vers la nature morte. 
EC On voit ensuite le tableau de Le Sueur performé dans ce qu'on pourrait appeler un tableau vivant dans sa forme plus habituelle. | fig. 3 |

ÉM Il y a trois moments où l'on voit le tableau vivant dans son ensemble: à l'extérieur, qui est en fait la cour de l'Université de Montréal. On ne le voit pas très longtemps. L'idée était de le voir presque incidemment, que la caméra ne fasse que passer devant le tableau figé. Puis le tableau a été filmé en studio avec un fond d'écran, ce dont je me suis servi de deux façons: d'abord, on voit l'écran vert, | fig. 4 | ce qui dénote l'artifice du studio; puis, avec un fond d'arbres présenté avec un effet spécial de vieux film. | fig. $5 \mid$ Cette allusion m'a parue intéressante, car le cinéma des premiers temps est un moment où il y a eu énormément de tableaux vivants. Ainsi, un des premiers usages du cinéma, ce n'est pas l'image animée, mobile, mais l'image fixe, et je voulais faire allusion à cette ironie.

EC Je pense qu'une des raisons pour lesquelles il y a autant de tableaux vivants dans le cinéma des premiers temps est que le médium du cinéma permet de mettre en scène la tension entre l'immobilité et le mouvement des figurant.es. Cette tension est beaucoup moins visible dans un médium comme la photographie. Par ailleurs, cette addition d'une nouvelle temporalité est en quelque sorte dépaysante et rend encore plus évidente l'idée que différentes temporalités se trouvent ici juxtaposées ou superposées.

ÉM En effet, c'était une des raisons de faire un tableau vivant: de jouer avec cette apparente immobilisation du temps et simultanément cette épaisseur temporelle, qui essaie de saisir qu'il y a des temps imbriqués les uns dans les autres.

Faire ce tableau vivant à l'extérieur avec les gens qui passent, qu'on voit sur leurs téléphones cellulaires, était une manière de jouer avec plusieurs temporalités. Mettre en studio les images truquées du cinéma des premiers temps sur le tableau vivant me permettait de jouer avec une autre temporalité. Ainsi, chaque fois se présentait comme une façon de réfléchir sur ce qu'est une transmission: qu'est-ce qui se transmet? comment? comment est-ce qu'on transmet un tableau? Il n'y a pas seulement l'original dans le musée. Il y a d'autres façons de transmettre un tableau, de lui faire traverser le temps. Il est à la fois le même et pas du tout le même. Quand on arrive enfin à voir l'original dans le film, comme on ne le voit que très tard, on devrait être un peu piégé par toutes les images qu'on a vues jusque-là. Cette vision de l'original prend d'autant plus d'intérêt qu'elle est imbriquée dans une temporalité, et qu'au lieu de partir de lui, on va vers lui, vers l'original, avec une sorte d'épaisseur temporelle.

MARION FROGER Pour revenirà la notion de tension: la tension du corps dans la posture du modèle a quelque chosequi ressemble un peu à la tension que l'on ressent quand on pose pour le tableau vivant, sauf qu'elle est beaucoup plus relâchée. J'avais posé pour un peintre-une fois-quand j'avais environ unevingtaine d'années. Alors que le temps de pose est plus long pour le modèle, on sait que ce n'est pas l'immobilité qui est importante, mais généralement ce quel'on dégage dans telle ou telle position. On doit tenir la pose pour qu'ilyait un minium de ressemblance, mais ce n'est pas là l'enjeu. Pour le tableau vivant, c'estvraiment l'idée d'une immobilitéà conserver. On est tellement tendu dans la pose qu'on 
Figure 1. Eustache Le Sueur (atelier de), Allégorie du ministre parfait, 1653 , huile sur toile, $85,0 \times 72,0 \mathrm{~cm}$. Dunkerque, Musée des Beaux-Arts de Dunkerque. Photo: Christian Jean, (c) RMN-Grand Palais / Art Resource, NY.

Figure 2. Éric Méchoulan (réalisateur), Une disparition. Enquête su un tableau de cheminée d'Eustache Le Sueur, Allégorie du ministre parfait (1653), $2017.26 \mathrm{~min}$.

[https://vimeo.com/212632352]. (arrêt: 4 min $58 \mathrm{~s}$.)
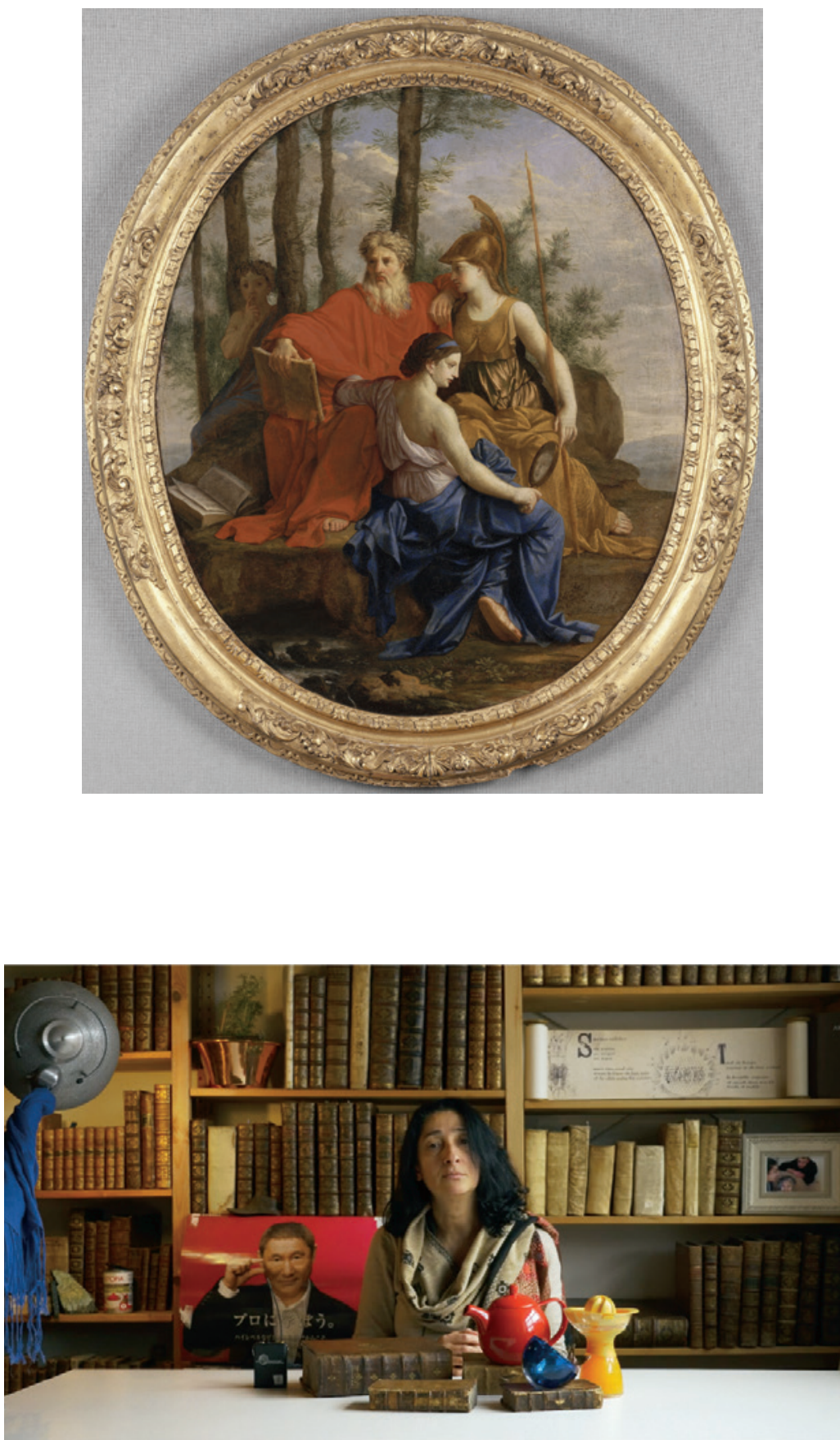
Figure 3. Éric Méchoulan (réalisateur), Une disparition. Enquête sur un tableau de cheminée d'Eustache Le Sueur, Allégorie du ministre parfait (1653), 2017. $26 \mathrm{~min}$.

https://vimeo.com/212632352]. (arrêt: 7 min 39s.)

Figure 4. Éric Méchoulan (réalisateur), Une disparition. Enquête sur un tableau de cheminée d'Eustache Le Sueur, Allégorie du ministre parfait (1653), $2017.26 \mathrm{~min}$.

[https://vimeo.com/212632352]. (arrêt: 14 min 24S.)

Figure 5. Éric Méchoulan (réalisateur), Une disparition. Enquête sur un tableau de cheminée d'Eustache Le Sueur, Allégorie du ministre parfait (1653), $2017.26 \mathrm{~min}$.

[https://vimeo.c-om/212632352] (arrêt: 14 min $42 \mathrm{~s}$.)
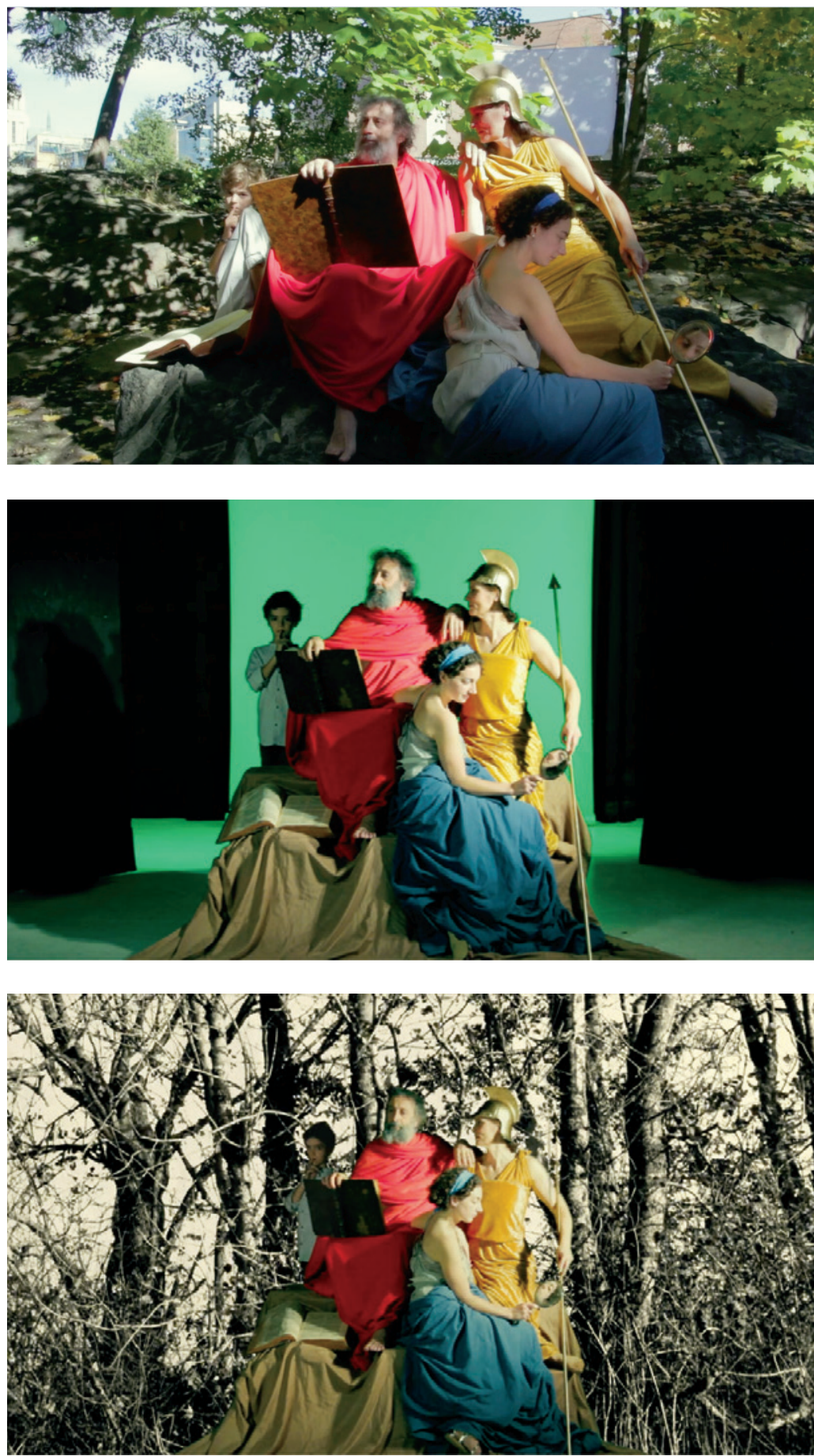
ne peut pas la tenir longtemps, même si elle est moins compliquée que celle qu'on avait devant le peintre. On est dans cette tension, disant «on vise l'immobilité, ce moment de suspension totale», eton saitqu'on n'yarrivera pas tous en même temps. Peut-être qu'un sourcil va vibrer ou que la respiration va sevoir.

EC Le tableau de Le Sueur réapparaît dans une autre sorte de tableau vivant, alors que Marion, cette fois-ci seule, dans le studio, tient un livre intitulé Le Classicisme français dont la page couverture reproduit le tableau de Le Sueur. | figs. 6-7| II y a ainsi jeu entre le tableau, le tableau vivant et l'espace de tournage.

ÉM Athéna faisant partie du tableau, montrant et lisant le texte sur son propre tableau, était une mise en abyme qui me semblait fort intéressante, d'autant plus que le tableau avait servi de photo de couverture à cet ouvrage sur le classicisme français.

MF Ce livre avait circulé pour que nous puissions bien vérifier la pose au moment de la prise des images. Et là il se retrouvait à l'intérieur lu par un personnage. J'avais la sensation d'avoir ramassé le livre du tournage pour le mettre dans l'espace du tableau.

EC Est-ce que vous trouvez que prendre part à un tableau vivant est un acte créatif? Est-ce que vous créez quel que chose, ou ne faites-vous qu'imiter?

ÉM Je réponds en tant que dix-septiémiste: l'opposition création / imitation ne fonctionne pas. Il n'y a pas de création qui ne soit pas imitation au $\mathrm{XVII}^{\mathrm{e}}$ siècle. Toute imitation est une re-création, donc une création. On pourrait en faire une spécificité de l'âge classique dans son rapport à l'antiquité. Toute imitation est de toute façon une manière de créer-et ici, par la distance ironique, par l'inadéquation des gestes qui ne sont pas exactement les mêmes, les couleurs non plus, dans l'économie générale du fond, des roches, des arbres...

MF C'est vrai qu'en faisant ce tableau vivant, je n'avais pas l'impression de refaire le tableau de Le Sueur, j'avais l'impression de faire notre tableau, c'est à dire, le tableau qu'Éric était en train de refaire, et qu'il était aussi en train d'inventer pour un film qui était encore à venir. Autant j'étais dans l'idée d'une démarche-il fallait que ce soit ressemblant pour que cette idée-là s'incarne, qu'elle ait du sens -, autant je savais que le but était de faire un film, donc un tout autre tableau, dans un tout autre contexte.

EC Marion, en tant que chercheuse en cinéma, pensez-vous avoir abordé le projet d'un point de vue différent?

MF Quand Éric m'a parlé de ce projet, je n'avais pas vu beaucoup de tableaux vivants, hormis ceux du cinéma des premiers temps et Passion de Godard (1982). Passion était une référence très importante pour moi, d'autant plus que dans ce film, Godard en profite pour faire un portrait du cinéma. Le tableau vivant est un prétexte pour parler du tournage d'un film, des rapports de pouvoir dans les équipes de tournage, du monde du cinéma en général. Ce qui m'avait le plus attirée dans le projet d'Éric, c'est qu'il y avait aussi peut-être cette idée de pouvoir faire, en filigrane, le portrait de la recherche, du monde académique. Les autres partenaires d'Éric sont en effet des collègues chercheurs, des professeurs; ce sont des étudiants du département d'histoire de l'art et d'études cinématographiques qui constituaient l'équipe technique. 
Il y avait aussi le fait qu'il ait fait ce film pour un colloque anniversaire. Pour fêter les vingt ans de la constitution du GRILH (Groupe de recherches interdisciplinaires sur l'histoire du littéraire), ses membres lui avaient demandé de faire une communication spéciale, et il avait opté pour un film qui demandait de rassembler du monde autour de lui. Tous ces éléments me renvoyaient au projet de Godard, qui était de faire des tableaux vivants et d'en profiter pour parler du cinéma comme milieu. Là, c'était Éric qui faisait un tableau vivant et pour ce faire, mobilisait des collègues, et parlait aussi de l'université et du monde des chercheurs en y puisant toutes ses ressources humaines et matérielles. Par exemple, on peut percevoir, dans la force d'éloquence de Djemaa qui rend possible le fait de voir, dans une théière et un presse-agrumes, des personnages allégoriques, aussi la performance de la professeure qu'elle est. Djemaa joue très bien son didactisme, elle ne fait pas que nous expliquer l'allégorie qu'elle dresse devant elle. Avec le plus grand des sérieux, et une pointe de malice, elle dispose savamment devant elle une théière, un presse-agrumes et un cendrier avec une cuiller, comme si cette reconstitution allait de soi. Et derrière elle, bien sûr, il y a un mur de livres. J'y vois une allusion au travail du chercheur, une mise en scène de ce que c'est que d'être un littéraire qui s'intéresse aux images, et de faire un film qui s'intéresse aux images, quand il faut inventer des images autant que les étudier. On pourrait aussi voir le rapport que l'on peut avoir en tant que chercheur et professeur aux objets que l'on étudie, qui est mis en scène parfois de façon très humoristique-par exemple, quand Éric lui-même passe dans son objet [Éric Méchoulan incarne le Conseil dans les tableaux vivants].

Ém Le fait de filmer le tableau vivant dans la cour de l'Université de Montréal a ajouté en effet à cette dimension.

EC En le filmant là, vous investissez le lieu d'une façon différente et vous prenez le risque de perdre le contrôle de la lumière, des bruits environnants, etc., que l'on pourrait avoir dans un studio-ou, si l'on présentait un tableau vivant au XIX ${ }^{\mathrm{e}}$ siècle, dans un salon ou un théâtre.

ÉM En partie, je trouvais cela amusant d'être dehors, mais il me semblait aussi que faire un tableau vivant dehors, mais aussi dans un studio, était une manière de réfléchir à des questions de lieu: qu'est-ce qu'un lieu? quel est le rapport entre l'intérieur et l'extérieur? pourquoi est-ce qu'on voit toujours les tableaux à l'intérieur d'un bâtiment et pas dehors? qu'est-ce que ça change?

Une des choses qui me paraissaient intéressantes historiquement parlant était d'inclure ce tableau dans une série-puisque c'était une série d'allégories-ce qu'on retrouve dans les entrées royales et les architectures éphémères, créées pour les entrées solennelles qui apparaissent depuis la fin du Moyen Âge jusqu'aux $\mathrm{XVI}^{\mathrm{e}}$ et $\mathrm{XVII}^{\mathrm{e}}$ siècles. On construisait des scènes où apparaissaient des personnages, parfois avec des banderoles, des étiquetages qui les identifiaient comme étant telle ou telle vertu, par exemple, ou parfois pour dire quelque chose au roi ou au prince qui passait. Si ces scènes ne représentaient pas nécessairement un tableau, elles faisaient tableau.

La représentation par Le Sueur d'une allégorie du bon gouvernement cadre tout à fait dans ces allégories politiques. Et la Fronde (1648-1653) est un moment intéressant dans ce contexte. C'est probablement autour de ce moment-là que les entrées solennelles déclinent, tant en nombre qu'en déploiement fastueux. 


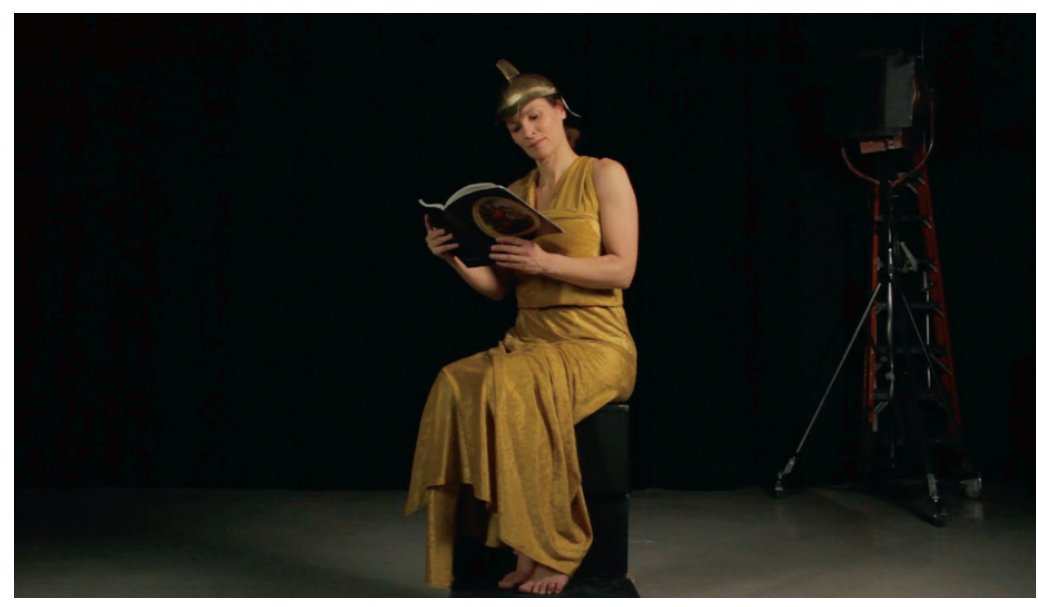

Figure 6. Éric Méchoulan (réalisateur), Une disparition. Enquête sur un tableau de cheminée d'Eustache Le Sueur, Allégorie du ministre parfait (1653), $2017.26 \mathrm{~min}$.

[https://vimeo.com/212632352]. (arrêt: 13 min 05s.)

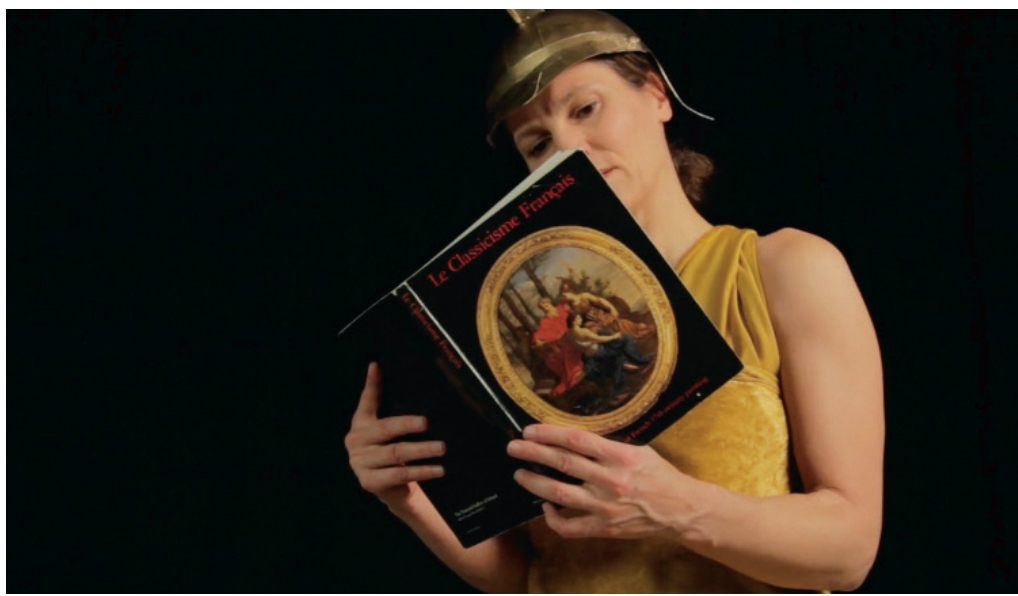

Figure 7. Éric Méchoulan (réalisateur), Une disparition. Enquête sur un tableau de cheminée d'Eustache Le Sueur, Allégorie du ministre parfait (1653), $2017.26 \mathrm{~min}$

[https://vimeo.com/212632352]. (arrêt: 13 min 19s.) 
D'un point de vue politique, les entrées solennelles sont vues comme des confirmations réciproques des liens entre la cité et le roi ou le prince. On échange clés, compliments et cadeaux, et on confirme que le pouvoir royal est le pouvoir royal, que le pouvoir de la cité est le pouvoir de la cité, et que tout fonctionne dans une grande harmonie. Mais les entrées se restreignent à partir des années 1620-1630, après certaines entrées de Louis XIII dans des pays protestants. C'étaient des pays en guerre, que l'on avait matés. Il ne s'agissait plus d'harmonie, mais de pouvoir, et ce phénomène s'est accru avec Louis xiv. La Fronde est un moment de bascule. Il me semblait important d'en parler avec ce tableau très typique qui s'inscrit dans la Fronde, un moment où on cesse de produire ces manières de faire tableau du bon gouvernement pour toute la population de la cité. On sort de l'espace public, donc de l'extérieur pour rentrer dans espace intérieur du petit tableau de cheminée. C'est très symptomatique au point de vue historique.

Par ailleurs, le tableau vivant tel qu'il s'institue à la fin du xvıII ${ }^{\mathrm{e}}$ siècle s'articule d'abord à cette capacité dans les entrées de faire tableau, mais cette fois-ci sur une scène qui existait déjà dans le théâtre. Il s'agissait d'un théâtre très classique, très hiératique, un théâtre de poses, de gestes codés et exécutés très lentement. Si le cinéma des premiers temps fonctionne tellement sur des tableaux vivants, c'est parce qu'ils reproduisent aussi des éléments théâtraux qui étaient joués, parfois par des amateurs ou parfois par des professionnels, qui euxmêmes reprenaient un modèle pictural.

EC Dans le film, la narratrice déclare: «les peintres ne peignent pas des corps, mais des forces». On le sent ici. On sent une sorte d'énergie coincée, une mise en réserve de cette force dans le tableau vivant qui a besoin de sortir. On ne la sent pas sur l'arrêt sur image autant que quand le film tourne.

MF Ça fonctionne grâce à la magie du cinéma et de la mise en scène. Dans la scène de la théière, il a fallu penser à ce cadrage, aux liens entre les gestes, à l'intonation et au texte qui devait être dit au moment où Djemaa devait poser tel ou tel objet. Dans le film, le jeu de couleurs est là pour ramener l'intensité d'une force, qui a pu être à la base des rapports de pouvoirs qui s'incarnent dans ce tableau, ou l'intensité d'une croyance, peut-être dans la perfection possible d'un gouvernement. Le film est une succession d'idées, d'explorations un peu ludiques et un peu magiques, soit plusieurs manières de redonner corps, de redonner vie à cette intensité à laquelle prétend le tableau, un tableau qui dit: je ne suis pas seulement une image, je suis la composition intense des idées ou des forces qui prévalaient à ma naissance.

Ém J'ai essayé de dire que dans le tableau vivant, ce ne sont pas des corps qui apparaissent, mais des forces. Même chose dans la peinture. Il n'y a pas de solution de continuité, ou tout du moins, pas là où on la mettrait. Ce n'est pas un déplacement de représentations de taches colorées sur une surface plane qui passeraient à des corps tridimensionnels vivants, respirants. C'est plutôt une translation de forces. Dans un sens, au moins, le tableau vivant permet peutêtre de mieux voir ou ressentir les forces présentes dans le tableau. 